\title{
Exciton Gas Compression and Metallic Condensation in a Single Semiconductor Quantum Wire
}

\author{
B. Alén, ${ }^{1, *}$ D. Fuster, ${ }^{1}$ G. Muñoz-Matutano, ${ }^{2}$ J. Martínez-Pastor, ${ }^{2}$ Y. González, ${ }^{1}$ J. Canet-Ferrer, ${ }^{2}$ and L. González ${ }^{1}$ \\ ${ }^{1}$ IMM, Instituto de Microelectrónica de Madrid (CNM, CSIC), Isaac Newton 8, 28760 Tres Cantos, Madrid, Spain \\ ${ }^{2}$ ICMUV, Instituto de Ciencia de Materiales, Universidad de Valencia, P.O. Box 22085, 46071 Valencia, Spain
}

(Received 29 November 2007; published 8 August 2008)

\begin{abstract}
We study the metal-insulator transition in individual self-assembled quantum wires and report optical evidence of metallic liquid condensation at low temperatures. First, we observe that the temperature and power dependence of the single nanowire photoluminescence follow the evolution expected for an electron-hole liquid in one dimension. Second, we find novel spectral features that suggest that in this situation the expanding liquid condensate compresses the exciton gas in real space. Finally, we estimate the critical density and critical temperature of the phase transition diagram at $n_{c} \sim 1 \times 10^{5} \mathrm{~cm}^{-1}$ and $T_{c} \sim 35 \mathrm{~K}$, respectively.
\end{abstract}

DOI: 10.1103/PhysRevLett.101.067405

PACS numbers: 78.67.Lt, 71.30.+h, 71.35.-y

The so-called metal-insulator transition in photoexcited semiconductor quantum wires (QWRs) has motivated many experimental and theoretical investigations in the last years [1-10]. As known from bulk semiconductors, the insulating exciton gas becomes unstable at high carrier densities and transforms into a many body conducting state. Yet, in certain semiconductor systems, this transition occurs through condensation of the exciton gas into a metallic electron-hole liquid (EHL), while in others, the exciton gas is completely ionized into a metallic electronhole plasma (EHP). Despite their common metallic character, both states have very different physical properties. An EHL, like any other liquid, is characterized by a well defined interface with its surroundings and definite equilibrium density of $e$ - $h$ pairs [11,12]. Since the equilibrium density is constant, an increment of the excitation increases the size of the crystal volume occupied by the liquid but does not change its internal properties. On the contrary, an EHP is formed by unbound $e-h$ pairs which fill the entire excited volume. In this case, a more intense excitation leads to an increase of the EHP density and eventually to the disappearance of the excitons at the so-called Mott critical density. The existence of 1D electron-hole plasmas in semiconductor QWRs has been evidenced in optical experiments performed both, in QWR ensembles [1,2] and single QWRs [4,5]. However, the observation of electron-hole liquids in this kind of systems has been until know much more elusive. In this framework, in the following we shall present our results obtained in individual InAs/InP self-assembled QWRs and demonstrate that the metal-insulator transition can occur in this system through compression of the exciton gas and formation of a metallic liquid condensate, and not only through ionization of the exciton gas into a plasma as usually reported.

The isolated InAs nanowires studied here have been obtained by self-assembling methods on $\operatorname{InP}(001)$ and present a large in-plane aspect ratio with typical lengths exceeding more than 10 times their average width $w \sim$ $20 \mathrm{~nm}$ as shown in Fig. 1. More details about the growth procedure, overall emission properties and confocal optical setup can be found elsewhere [13,14]. At low temperatures, their ground state emission energy at $\sim 0.8 \mathrm{eV}(1.55 \mu \mathrm{m})$ implies an estimated QWR height after capping of $h \sim$ $3.3 \mathrm{~nm}$. [15] This small cross-sectional area, $w \times h$, leads to large subband energy spacings in both, the conduction $(>30 \mathrm{meV})$, and valence bands $(>10 \mathrm{meV})$, and ensures that nonequilibrium carriers in these QWRs are effectively confined in one dimension.

At $5.5 \mathrm{~K}$ and low excitation power, the single QWR photoluminescence spectrum shown in Fig. 1 consists of several narrow peaks whose linewidths, in the range of $\sim 0.3-0.5 \mathrm{meV}$, indicate a very good size uniformity [4,5]. Because of their elongated shape, the single QWR emission is slightly polarized along the QWR axis (1-10 crystal direction) yielding a polarization anisotropy ratio $\theta=$ $\left(P_{0}-P_{90}\right) /\left(P_{0}+P_{90}\right) \sim 23 \%$ [15]. The asymmetric confinement also enhances the anisotropic electron-hole exchange interaction (AEI) producing a relatively large polarization splitting of the neutral exciton line $\left(X^{0}\right)$ at $0.8325 \mathrm{eV}$ [16]. This splitting is absent when two electrons

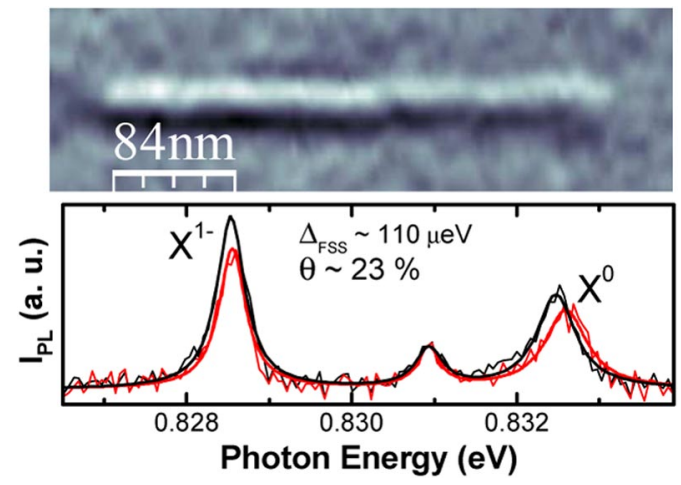

FIG. 1 (color online). Top panel: Atomic force micrograph of an uncapped sample showing an isolated self-assembled InAs/ InP QWR. Bottom panel: $\mu$ PL spectra of a single QWR obtained at $5.5 \mathrm{~K}$ with linear polarizations selected along (black lines) and across (red lines) the QWR axis. 
and one hole bond together into a negatively charged exciton or trion $\left(\mathrm{X}^{1-}\right)$ as it can be observed for the main emission line at $0.8285 \mathrm{eV}$ in Fig. 1. Narrow spectral features with similar splittings and polarization behavior are systematically observed in our spectra at low excitation power. Weaker resonances, like the one detected in this case at $0.8309 \mathrm{eV}$, appear occasionally and will be tentatively assigned to the emission from localized exciton states whose anisotropy and splitting cannot be resolved due to their more symmetric wave functions.

The presence of negative trions in our spectra is not surprising given the rather high $n$-type residual doping of InP layers grown by conventional molecular beam epitaxy methods. We have estimated $N_{D} \sim 1 \times 10^{16} \mathrm{~cm}^{-3}$ by Hall resistance measurements implying an average occupation of at least two spectator electrons per QWR in absence of light. This fact is of central importance in our analysis since the emission line shape of the trion resonance strongly depends on its lateral extension and therefore on the size of the crystal volume occupied by the exciton gas as explained below. This dependence can be established following the formulation based on earlier theoretical work [17] of A. Esser et al [18] which, few years ago, studied the emission line shape of singly charged excitons in quantum wells (QWs). We have adapted here their results and considered a thermal distribution of 1D negative trions with mass $M_{T}=2 m_{e}+m_{h h}$ and energy $E_{T}+\hbar^{2} K_{0}^{2} / 2 M_{T}$. The emission line shape is given by [18]

$$
P_{T}(\hbar \omega) \propto\left|M\left(K_{0}\right)\right|^{2} \exp \left(-\frac{\epsilon}{k_{B} T} \frac{m_{e}}{M_{X}}\right) \frac{1}{\sqrt{\epsilon}},
$$

where $M_{X}=m_{e}+m_{h h}$ is the exciton mass, $\boldsymbol{\epsilon}=$ $E_{T}-\hbar \omega=\frac{\hbar^{2} K_{0}^{2}}{2 m_{e}} \frac{M_{X}}{M_{T}}$ results from the energy conservation, and the optical matrix element $M\left(K_{0}\right)$ is calculated separately from

$$
M\left(K_{0}\right)=\int_{-\infty}^{\infty} d x_{2} \Psi_{X^{1-}}\left(x_{1}=0, x_{2}\right) \exp \left(-i K_{0} x_{2} \frac{M_{X}}{M_{T}}\right),
$$

being $x_{i}$ the position of electron $i$ relative to the hole and $x_{1}=0$. Equation (1) can be evaluated analytically for the special case where the trion wave function, $\Psi_{X^{1-}}\left(0, x_{2}\right)$, is given by a Gauss shape reducing Eq. (2) to a simple Fourier transform in momentum space. The resulting line shapes have been depicted in Fig. 2(a) for three different Gaussian lengths, $a_{1 D}^{T}$, and constant temperature, $T=10 \mathrm{~K}$. As it can be observed, the more localized is the trion in real space, the longer extends its photoluminescence towards low energies, $\hbar \omega<E_{T}$. We have applied this scheme to our spectra by removing the inverse square root singularity from Eq. (1) in account of the finite trion lifetime $\left(\hbar / \Gamma_{T}\right)$. Thick solid lines in Fig. 2(b) represent the best fitting curves obtained varying $a_{1 D}^{T}$ to describe the power evolution of the trion resonance identified before at $0.8285 \mathrm{eV}$. It must be noted that the line shape evolution can be cached
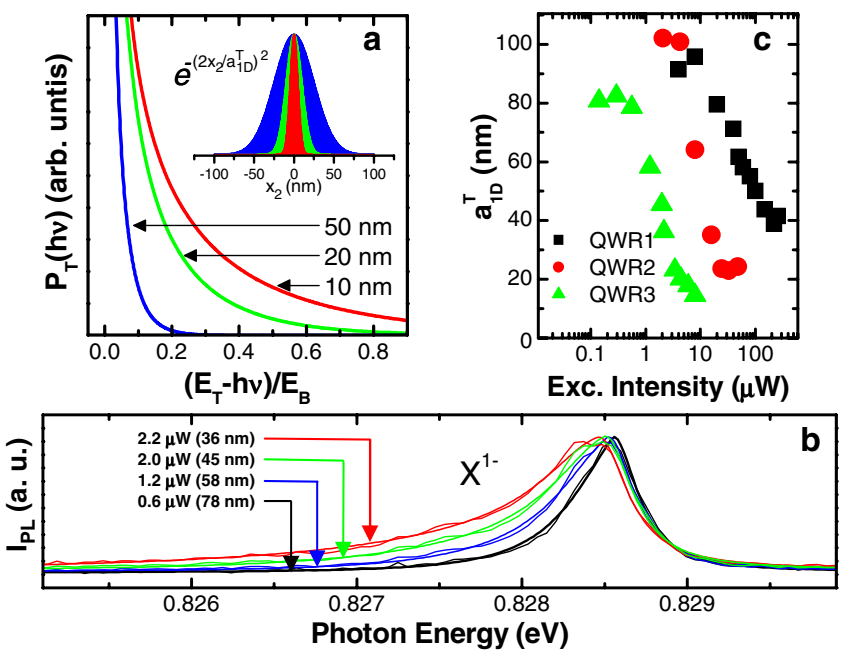

FIG. 2 (color online). (a) Evolution of the low energy emission tail calculated using Eq. (1) for charged excitons with different extent in real space ( $E_{B}$ stands for the trion binding energy). (b) The increasing asymmetry of the experimental trion resonance (thin solid lines) can be fitted to the model (thick solid lines). (c) Excitation power evolution of the trion wave function length, $a_{1 D}^{T}$, for different QWRs.

by the model meaning that the exciton trion wave function is being apparently compressed in real space upon increase of the excitation intensity. To the best of our knowledge, such observation has not been reported before in confined systems of any dimensionality.

The examination of the single QWR spectrum in a broader range can give us more information about the trion wave function shrinkage. Increasing the laser power, a relatively broad band appears at the low energy side of the spectrum, as depicted in Figs. 3(a) and 3(b) for two different single QWRs. At the same time, $a_{1 D}^{T}$ diminishes from $\sim 100 \mathrm{~nm}$ to $\sim 20 \mathrm{~nm}$ as shown in Fig. 2(c). The broad emission band line shape evolution can be described using a 1D single band momentum conserving scheme with constant matrix elements just as expected from the direct recombination of $e-h$ pairs at the renormalized band gap edge. [1] The thick solid lines in Figs. 3(a) and 3(b) are numerical fits obtained varying slightly the band gap energy, $E_{g}^{i}$, broadening parameter, $\Gamma_{g}$, and carrier temperatures, $T_{e(h)}$, and keeping fixed the chemical potential energy, $\mu_{e}+\mu_{h}$, at the trion peak position. The spectral analysis also allows the direct determination of the 1D carrier density, $n_{1 D}$, from [19] $E_{T}-E_{g}^{i}=E_{\mathrm{fe}}+E_{\mathrm{fh}}=$ $\frac{\pi^{2} \hbar^{2} n_{1 D}^{2}}{8 m_{e}}\left(1+m_{e} / m_{h}\right)$ which can be used to discriminate between a condensed phase and a plasma as explained below.

The results depicted in Fig. 3 represent a solid experimental evidence of the formation of an EHL in our system. At low excitation powers, an $e-h$ pair captured into the QWR can form a neutral exciton or, with the aid of an spectator electron, a negative trion. According to our results, the binding energy of the negative complex is around 


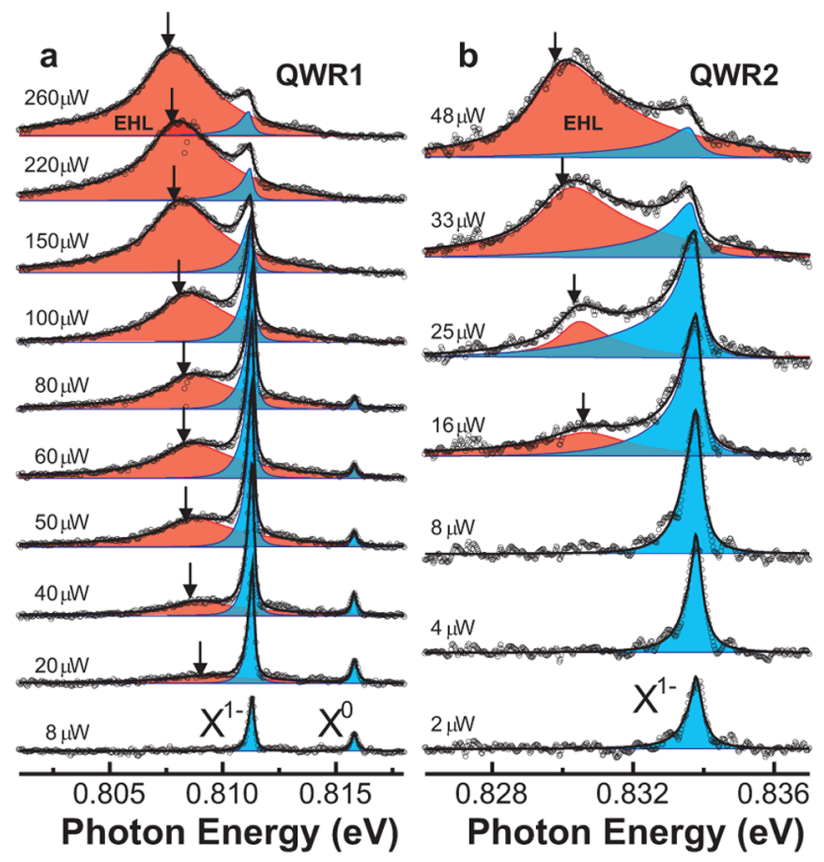

FIG. 3 (color online). (a) and (b) Emission spectra of different single QWRs upon increase of the excitation power. The line shape analysis reveals that the insulating excitonic gas (high energy peak) condensates into a metallic electron-hole liquid phase (low energy band). Down arrows stand for the position of the renormalized band edge in each spectrum.

3 to $5 \mathrm{meV}$ greater than the neutral one, and therefore trions will prevail while excess electrons remain available [20]. Above a certain excitation density, the overlap between individual exciton complexes is such that many body excitations develop in the nonequilibrium carrier ensemble. At this point, several spectral features reveal the presence of two spatially separated phases comprising an excitonic gas and an EHL. First, the excitonic emission peaks coexist with the band edge luminescence in a wide excitation window. Only when the latter is fully developed, the trion luminescence gets appreciably quenched, as observed in Fig. 3. Second, as expected from a liquid phase with constant internal properties, neither the position, nor the shape of the band edge emission changes significantly during the crossover. Indeed, despite the tenfold (QWR1) and threefold (QWR2) increase of the excitation intensity, and hence of the number of carriers, $n_{1 D}$ stays essentially constant, and only varies between 2.5 and $2.9 \times 10^{5} \mathrm{~cm}^{-1}$, and between 2.7 and $3.0 \times 10^{5} \mathrm{~cm}^{-1}$, respectively. Our results suggest a dynamical situation where after initial drop nucleation, the larger excitation increases the volume of the crystal occupied by the liquid inducing the compression of the exciton gas against the QWR edges as discussed above.

If we increase the excitation power further, new spectral features appear in the tail of the EHL band. This is the case of the semilogarithmic plot of the spectrum of QWR1 excited with $800 \mu \mathrm{W}$ and shown in Fig. 4(a). We tentatively assign the low energy shoulder that appears at high
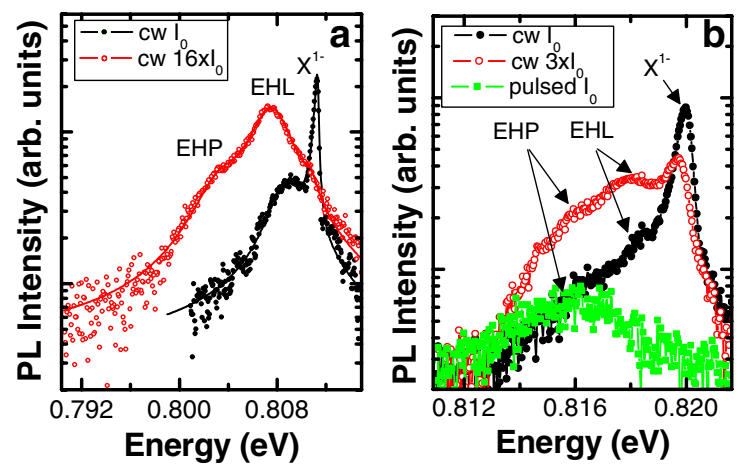

FIG. 4 (color online). The panels show the emission spectra of two different QWRs obtained using the excitation conditions indicated in each legend. In all cases the excitation wavelength was $980 \mathrm{~nm}$ and $I_{0}=50 \mu \mathrm{W}$.

excitation power to the formation of an electron-hole plasma (EHP) in the QWR. The same occurs for the nanowire represented in the adjacent figure where we used moderate excitation with a $980 \mathrm{~nm} 40 \mathrm{MHz}$ pulsed laser (40 ps pulsed duration) to deplete completely the excitonic and EHL emission and leave only the EHP band. We have observed the same behavior tuning the excitation energy of our Ti:sapphire laser from 1000 to $865 \mathrm{~nm}$. Only excitation well below the InP gap allowed the observation of sharp exciton peaks and distinct EHL features in qualitative agreement with previous work done in highly excited direct gap semiconductors [21].

The temperature dependence of the single QWR spectrum shown in Fig. 5 corroborates our hypothesis. The presence of strong quantum correlations within the many body ensemble is the main responsible of the appearance of a thermodynamically stable liquid phase instead of an electron-hole plasma in our system. Therefore, once the condensation has occurred, to extract an electron-hole pair out of the condensed phase, some energy, $E_{L}$, must be provided [12]. This evaporation mechanism must be considered to explain the evolution of the spectra shown in Fig. 5(a). Contrarily to the behavior expected for a metallic plasma, above $\sim 25 \mathrm{~K}$ the liquid begins to evaporate and the system returns to its gaseous state. Furthermore, at the turning point, the reduction of the integrated intensity of the EHL band and the enhancement of the trion emission peak evolve with the same rate. The value extracted from the slope of the integrated intensity, $E_{L} \sim 8 \mathrm{meV}$, fairly agrees with the energy splitting observed in most QWRs between the exciton peak $\left(X^{0}\right)$ energy and the onedimensional band edge position. Although the dynamic range of the fit is small, this could indicate that the evaporation of the liquid is an ambipolar process followed by the trion formation at a later step.

Our results can be summarized in a typical phase diagram for a metallic EHL as shown in Fig. 5(c) [12]. At low temperatures $(T \leq 20 \mathrm{~K})$ and moderate carrier densities $\left(n_{1 D} \leq 1 \times 10^{4} \mathrm{~cm}^{-1}\right)$, the system behaves as a diluted excitonic gas $(G)$ dominated by Coulomb interactions 

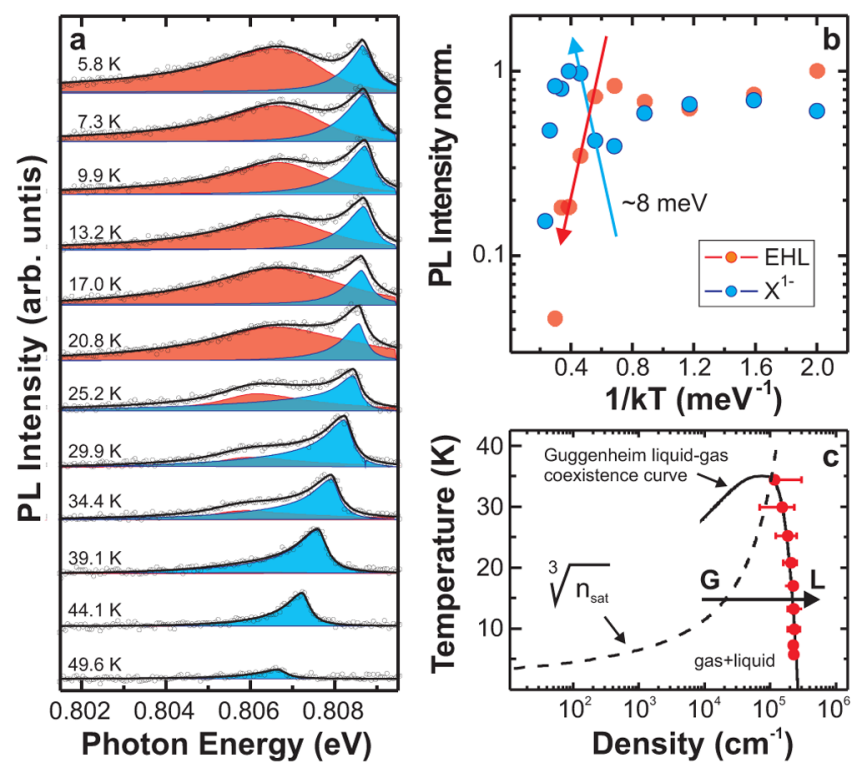

FIG. 5 (color online). (a) Evolution of the single QWR emission spectrum with temperature. The excitation intensity was chosen at $5.8 \mathrm{~K}$ to make visible both the excitonic and EHL contributions. (b) Excitonic and band edge integrated intensities (normalized) as obtained from the line shape fits. (c) Schematic phase diagram for the metal-insulator crossover in our system. The solid line represents the liquid-gas coexistence curve which better describes our experimental data (solid circles). The dashed line shows the evolution of the saturated gas density deduced roughly from the bulk InAs expression $n_{\text {sat }}=2\left(\frac{2 \pi m_{e}^{*} k_{B} T}{h^{2}}\right)^{3 / 2} e^{\left(-E_{L} / k_{B} T\right)}$ with $E_{L}=8 \mathrm{meV}$.

among only few particles. As the carrier density approaches $\sim 10^{5} \mathrm{~cm}^{-1}$, Coulomb correlations become increasingly important and, in some regions of the excited volume, the exciton gas condensate into metallic electronhole droplets. The coexistence region $(G+L)$ is limited by the equilibrium curves corresponding to the uniform gas (left branch) and the uniform liquid (right branch). It comprises the range of parameter values where a spatially uniform distribution is not possible, and there occurs a physical separation between well defined liquid and gaseous phases. The phase diagram shows a characteristic feature of the electron-hole liquid: the density decreases when the temperature increases towards the critical point following a Guggenheim-like liquid-gas coexistence curve $[22,23]$. Therefore, the comparison of this curve with the data extracted from the spectral line shape analysis allows the determination, within our experimental uncertainty, of the critical temperature, $T_{c}=35 \mathrm{~K}$, and critical density, $n_{c}=7.5 \times 10^{4} \mathrm{~cm}^{-1}$, for this single quantum wire.

Finally, we must discuss briefly how our results could be affected by the QWR morphology and impurity environment. In around one out of five studied QWRs, we have found that exciton localization induced by disorder of the confining potential prevents the formation of an EHL phase. As reported for other QWR systems, in such cases, the strong localization leads to a high power $\mu \mathrm{PL}$ spectrum characterized by radiative recombination of multiexciton complexes [4]. Their behavior is very different of the disorder-free QWRs presented in this work which show clear one-dimensional spectral features. Second, the impurity background has made possible the formation of negatively charged excitons and therefore the observation of the exciton trion compressional effect. Since the random impurity potential can pin the fermi level in different regions of the QWR, [24] weak localization of the electrons could make easier the initial liquid drop nucleation in our system, although, at present, its precise role remains an open question.

In conclusion, we have identified a novel behavior studying the metal-insulator transition in individual semiconductor nanowires. Our experimental results reveal that in this system this transition can occur through compression of the exciton gas and condensation in a metallic liquid phase and should motivate future theoretical and experimental investigation of these and related excitonic and correlated electron phenomena.

The authors acknowledge valuable discussions with Dr. Jose M. Llorens, Dr. Richard J. Warburton, and Dr. Khaled Karrai. Financial support by the Spanish MEC and CAM through projects Nos. TEC-2005-05781-C0301/03, NAN2004-09109-C04-01/03， S-505/ESP/000200 and CSD2006-0019, and by the European Commission through SANDIE Network of Excellence (No. NMP4CT-2004-500101) is also gratefully acknowledged.

*benito@imm.cnm.csic.es

[1] R. Cingolani et al., Phys. Rev. Lett. 67, 891 (1991).

[2] C. Gréus et al., Europhys. Lett. 34, 213 (1996).

[3] R. Ambigapathy et al., Phys. Rev. Lett. 78, 3579 (1997).

[4] T. Guillet et al., Phys. Rev. B 68, 045319 (2003).

[5] M. Yoshita et al., Phys. Rev. B 74, 165332 (2006).

[6] T. Ihara et al., Phys. Rev. Lett. 99, 126803 (2007).

[7] S. DasSarma and D. W. Wang, Phys. Rev. Lett. 84, 2010 (2000).

[8] D. W. Wang and S. DasSarma, Phys. Rev. B 64, 195313 (2001).

[9] K. Asano and T. Ogawa, J. Lumin. 112, 200 (2005).

[10] P. Huai and T. Ogawa, J. Lumin. 119-120, 468 (2006).

[11] T. M. Rice, Solid State Physics (Academic, New York, 1977), Vol. 32.

[12] L. V. Keldysh, Contemp. Phys. 27, 395 (1986).

[13] D. Fuster et al., Nanotechnology 18, 035604 (2007).

[14] B. Alén et al., Appl. Phys. Lett. 89, 233126 (2006).

[15] B. Alén et al., Appl. Phys. Lett. 78, 4025 (2001).

[16] A. Högele et al., Physica (Amsterdam) 21E, 175 (2004).

[17] B. Stébé et al., Phys. Rev. B 58, 9926 (1998).

[18] A. Esser et al., Phys. Rev. B 62, 8232 (2000).

[19] J. M. Calleja et al., Solid State Commun. 79, 911 (1991).

[20] B. Szafran et al., Phys. Rev. B 71, 235305 (2005).

[21] M. Nagai et al., Phys. Rev. Lett. 86, 5795 (2001).

[22] L. M. Smith and J. P. Wolfe, Phys. Rev. B 51, 7521 (1995).

[23] E. A. Guggenheim, J. Chem. Phys. 13, 253 (1945).

[24] A. L. Efros, Solid State Commun. 70, 253 (1989). 